\title{
(R)-a-Aminoadipic acid: an interesting chiral pool building block
}

\author{
Amina Sadiq and Norbert Sewald * \\ Department of Chemistry, Bielefeld University, Universitätsstraße 25, 33615 Bielefeld, Germany \\ E-mail: Norbert.sewald@uni-bielefeld.de
}

Dedicated to Prof. Ferenc Fülöp on the occasion of his $60^{\text {th }}$ birthday

\begin{abstract}
$(R)$ - $\alpha$-Aminoadipic acid is available on a large scale by enzymatic cleavage from cephalosporin $\mathrm{C}(\mathrm{CephC})$ in the production of 7-aminocephalosporanic acid (7-ACA). It can be converted into other interesting enantiomerically pure compounds, e.g. derivatives of $(R)$-pipecolic acid $(R$ piperidine-2-carboxylic acid), ( $R$ )-6-oxopiperidine-2-carboxylic acid, $(R)$-1,2,3,4-tetrahydropyridine-2(2H)-carboxylates, and other compounds obtained by further conversions of these products.
\end{abstract}

Keywords: $(R)$ - $\alpha$-Aminoadipic acid, $(R)$-Pipecolic acid, Vilsmeier-Haack reaction

\section{Introduction}

Amino acids are appreciated by synthetic chemists as inexpensive and versatile enantiomerically pure building blocks. Besides in the synthesis of peptides and peptidomimetics, amino acids found widespread application as starting materials or even catalysts in the synthesis of a broad range of compounds. ${ }^{1}(R)$-Configured amino acids do not occur in Nature as frequently as their $(S)$-configured enantiomers. $(R)$-Amino acids exert conformational bias, when they are incorporated e.g. in cyclic peptides. ${ }^{2}$ Moreover, the sense of chirality also renders them interesting starting materials for synthesis.

$(R)$ - $\alpha$-Aminoadipic acid $\mathbf{1}$ is a constituent of penicillin $\mathrm{N}$ and cephalosporin $\mathrm{C}$. In the semisynthesis of other penicillin and cephalosporin derivatives $(R)$ - $\alpha$-aminoadipic acid is first cleaved by chemical or enzymatic means to give 6-aminopenicillanic acid (6-APA) and 7aminocephalosporanic acid (7-ACA), resp. 6-APA and 7-ACA are then acylated to give different penicillins and cephalosporins. In the case of cephalosporin $\mathrm{C}$ cleavage by cephalosporin acylase provides $(R)$ - $\alpha$-aminoadipic acid as a side product in large quantities. ${ }^{3}$

We therefore embarked on a project to explore, how the enantiomerically pure $(R)$ - $\alpha$-aminoadipic acid could be used as a building block in the preparation of other compounds. Besides the 
known conversion into the $\delta$-lactam 2, we envisaged the transformation into benzyl 1benzyloxycarbonyl-5-formyl-1,2,3,4-tetrahydropyridine-2-carboxylate $\mathbf{3}$ and $(R)$-pipecolic acid 4.

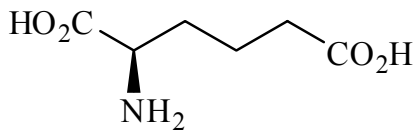

1

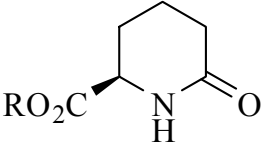

2a $\mathrm{R}=\mathrm{CH}_{3}$ 2b $\mathrm{R}=\mathrm{C}_{2} \mathrm{H}_{5}$

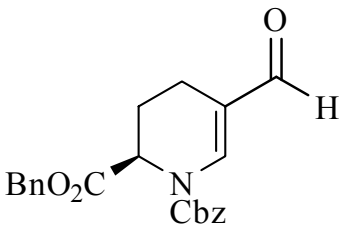

3

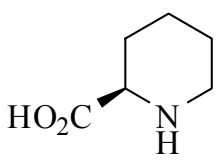

4

Pipecolic acid, ${ }^{4}$ also known as homoproline or piperidine-2-carboxylic acid, is a naturally occurring cyclic amino acid which displays interesting and potent biological activities. The presence of $(S)$-pipecolic acid in many biologically active natural products such as the immunosuppressant FK 506, ${ }^{5}$ rapamycin, ${ }^{6}$ the anticancer agent $\mathrm{VX} 710{ }^{7}$ and the antifungal antibiotic sandramycin. ${ }^{8}(R)$-Pipecolic acid occurs in the central nervous system of mammals to control the synaptic transmission and is also a well-known intermediate in the synthesis of piperidine alkaloids. Furthermore, it is a constituent of the histone deacetylase (HDAC) inhibitor apicidin. ${ }^{9,10}$ HDAC inhibitors hold promise as anticancer therapeutics. ${ }^{11}$ Moreover, $(R)$-pipecolic acid has also been recently employed as a catalyst for asymmetric Mannich reactions. ${ }^{12}$

Like proline, pipecolic acid is also a valuable building block for the synthesis of conformationally constrained peptides. ${ }^{13}$ Hence, a convenient access to $(R)$-pipecolic acid is of importance because it is a key constituent of bioactive molecules, a useful building block for asymmetric synthesis and a versatile organocatalyst.

\section{Results and Discussion}

Having access to $(R)$ - $\alpha$-aminoadipic acid from the industrial process, we focused on the synthesis of $(R)$-pipecolic acid 4. Enantiomerically pure pipecolic acid has previously been available by different procedures. ${ }^{14}$ We developed a new and simple synthesis of $(R)$-pipecolic starting from $(R)$ - $\alpha$-aminoadipic acid. The most useful amino protecting groups are the carbamates, which are extensively used for the synthesis of amino acids and other natural products. In particular, benzyl carbamates are useful for amino acid synthesis as they are resistant to a variety of reagents, can readily be cleaved and minimize the racemization of amino acids. In the course of our studies, we protected the amino group as well as selectively the $\alpha$-carboxylic group of $(R)$ - $\alpha$-aminoadipic acid with benzyl based protective groups because a final deprotection step removes all protective groups in one step. 

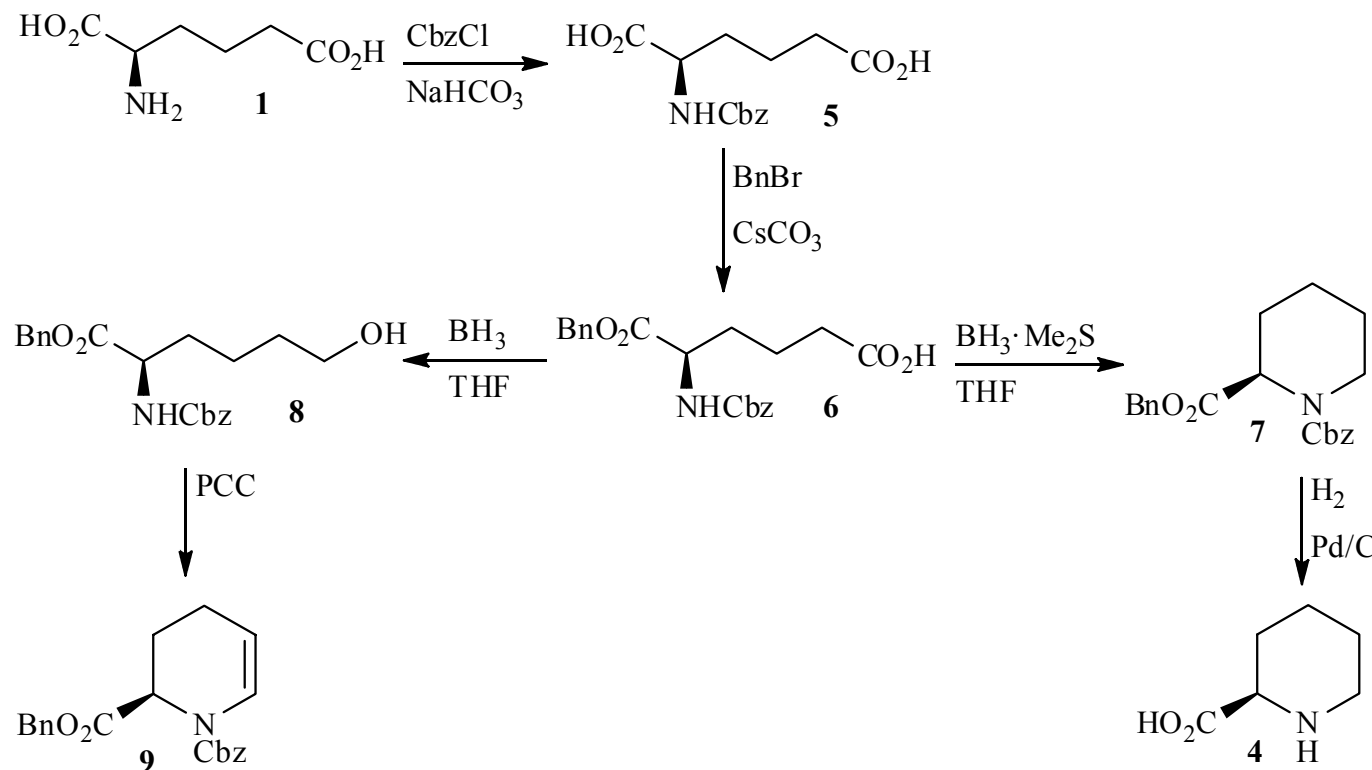

Scheme 1. Protection of $(R)$ - $\alpha$-aminoadipic acid 1, synthesis of $(R)$-pipecolic acid 4, and of the dehydro derivative 9.

The amino group of $(R)$ - $\alpha$-aminoadipic acid $\mathbf{1}$ was protected with the $\mathrm{Cbz}$ group to give urethane 5 in $89 \%$ yield. ${ }^{15}$ This was then selectively esterified with a benzyl ester moiety at the $\alpha$-position to give 6 in $40 \%$ yield according to a literature procedure. ${ }^{16}$ The carboxy group of intermediate 6 was then subjected to reductive cyclization on treatment with $\mathrm{BH}_{3} \cdot \mathrm{Me}_{2} \mathrm{~S}$ to prepare the protected $(R)$-pipecolic acid 7 in $63 \%$ yield. Hydrogenation of 7 over $\mathrm{Pd} / \mathrm{C}(10 \%)$ in the presence of a catalytic amount of $\mathrm{AcOH}$ afforded the crude product which was then purified by triturating with diethyl ether to furnish the desired product 4 in $88 \%$ yield (Scheme 1).

Reduction of ent-6 with borane - THF is reported to afford the corresponding alcohol ent-8 in $74 \%$ yield, which can be transformed into the dehydro derivative ent-9 in $46 \%$ yield. ${ }^{17} \mathrm{We}$ followed this procedure to convert 6 to 9 in $40 \%$ total yield and used the latter compound as a starting material for a Vilsmeier-Haack reaction. In the frame of this procedure, electron rich aromatic compounds or alkenes react with an iminium salt formed in situ from $N, N$ dimethylformamide and phosphorus oxychloride. Aldehyde 3 was obtained in $73 \%$ yield and shown to be enantiomerically pure (ee $>99 \%$ ) by chiral HPLC (Chirex $(S)$-tert.-leucine).

3 was subsequently used as an intermediate to synthesize various new derivatives of $(R)$ pipecolic acid. The Wittig reaction afforded product $\mathbf{1 0}$ where a vinyl group is introduced at C-5 of the pipecolic acid core (Scheme 2). Exemplarily, this reaction was carried out with the phosphorus ylid generated from methyl triphenylphosphonium bromide with $n$-BuLi at $-78{ }^{\circ} \mathrm{C}$ to afford the vinyl substituted product $\mathbf{1 0}$ in $60 \%$ yield. 


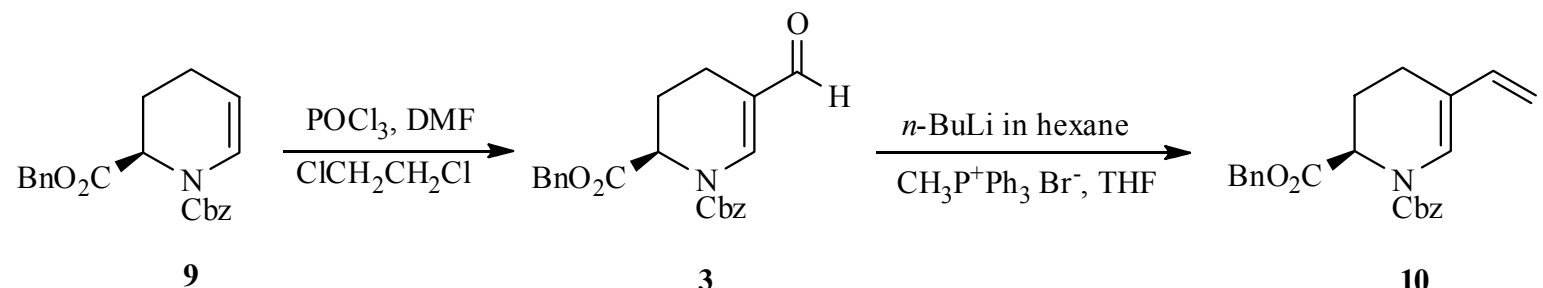

Scheme 2. Synthesis of formyl and vinyl derivatives of $(R)$-pipecolic acid.

$(R)$-6-Oxopipecolates 2 were prepared from $(R)$ - $\alpha$-aminoadipic acid by esterification and subsequent cyclization during Kugelrohr distillation in excellent yield according to a protocol described for (S)-6-oxopipecolic acid (ent-2). ${ }^{18}$ Compound $\mathbf{1 1}$ was formed in $70 \%$ yield upon reaction of $\mathbf{2}$ under Vilsmeier-Haack conditions as described for $\mathbf{3}$ (Scheme 3 ).

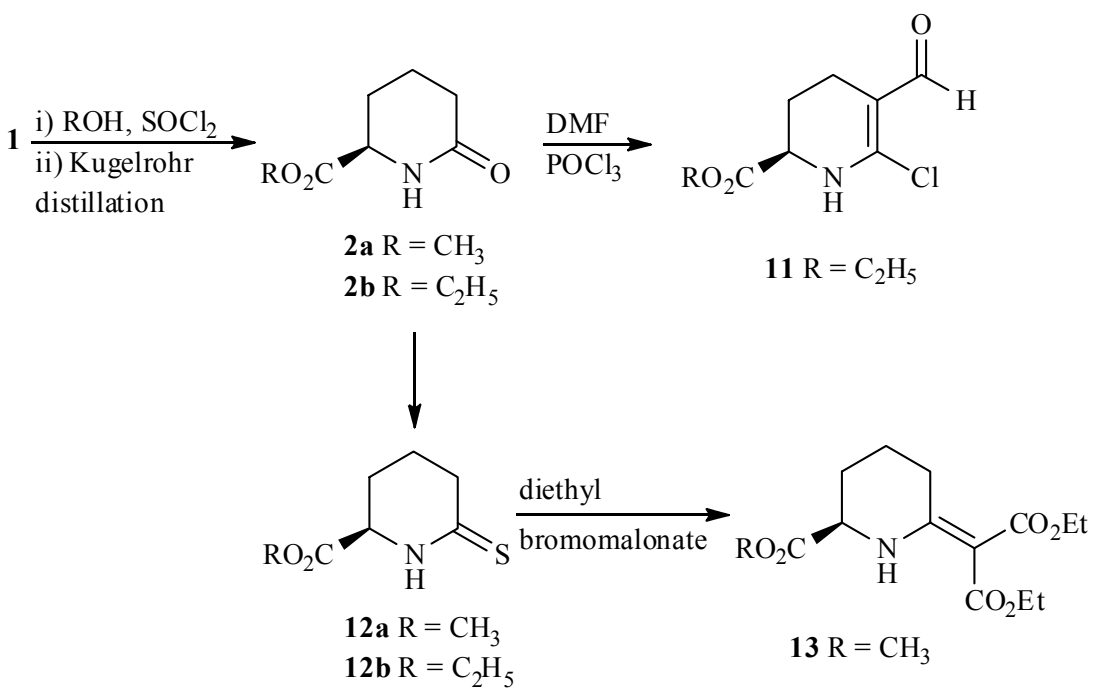

Scheme 3. Vilsmeier-Haack formylation of $\mathbf{2}$, thionation of $\mathbf{2}$ and further conversion.

Thiolactams $\mathbf{1 2 a} / \mathbf{b}$ were prepared in $80 \%$ and $56 \%$ yield, resp., by thionation of the corresponding $\delta$-lactams 2 with 1.8 equivalents of Lawesson's reagent like described previously for gluconolactam derivatives. ${ }^{19}$ 12a was further treated with diethyl bromomalonate in presence of triethyl amine to produce compound $\mathbf{1 3}$ in $72 \%$ yield.

In summary, a simple and completely stereoconservative access to $(R)$-pipecolic acid starting from $(R)$ - $\alpha$-aminoadipic acid has been developed. Furthermore, the synthesis of novel $(R)$ pipecolic acid derivatives by Vilsmeier-Haack reaction and further transformations of these products lead to interesting amino acids. 


\section{Experimental Section}

General. THF was dried over Na/benzophenone and DCM was dried over $\mathrm{CaH}_{2}$. Commercially available chemicals were purchased from Sigma-Aldrich. EtOAc was distilled before use. All reactions were carried out in oven-dried glassware with magnetic stirrers under an argon atmosphere. Flash chromatography was carried out using silica gel, particle size $0.035-0.070$ $\mathrm{mm}$. Specific rotation of synthesized compounds was recorded on a Jasco DIP-366 digital polarimeter. ${ }^{1} \mathrm{H}$ NMR spectra were recorded on a $500 \mathrm{MHz}$ Bruker DRX spectrometer in $\mathrm{CDCl}_{3}$ (unless otherwise stated) referenced relative to residual $\mathrm{CHCl}_{3}(\delta=7.26 \mathrm{ppm})$. Chemical shifts are reported in ppm and coupling constants in Hertz. ${ }^{13} \mathrm{C}$ NMR spectra were recorded on the same instrument $(125.7 \mathrm{MHz})$ with total proton decoupling.

EI and CI mass spectra (including EI accurate mass measurements) were recorded using an Autospec X magnetic sector mass spectrometer with EBE geometry (Vacuum Generators, Manchester, UK) equipped with a standard EI or CI source. Samples were introduced by push rod in aluminium crucibles if not otherwise noted. Ions were accelerated by $8 \mathrm{kV}$ in EI mode and $6 \mathrm{kV}$ in $\mathrm{CI}$ mode. accurate mass measurement (or EI/CI) experiments were performed using a Fourier Transform Ion Cyclotron Resonance (ESI- or MALDI-FT-ICR) mass spectrometer APEX III (Bruker Daltonik GmbH, Bremen, Germany) equipped with a 7.0 T, $160 \mathrm{~mm}$ bore superconducting magnet (Bruker Analytik GmbH - Magnetics, Karlsruhe, Germany), infinity cell, and interfaced to an external (nano)ESI or MALDI ion source. Nitrogen served both as the nebulizer gas and the dry gas for ESI. Nitrogen was generated by a Bruker nitrogen generator NGM 11. Argon served as cooling gas in the infinity cell and collision gas for $\mathrm{MS}^{\mathrm{n}}$ experiments.

$\boldsymbol{N}$-Benzyloxycarbonyl- $(\boldsymbol{R})$ - $\alpha$-aminoadipic acid; Cbz- $(\boldsymbol{R})$-Aad(OH)-OH (5). ${ }^{15} \mathrm{M} . \mathrm{p}=133-$ $135^{\circ} \mathrm{C}$; lit. ${ }^{15} \mathrm{~m} . \mathrm{p}=136-136.5^{\circ} \mathrm{C} .[\alpha]_{\mathrm{D}}{ }^{23}=+15.1(\mathrm{c}=1, \mathrm{EtOH} / 2 \mathrm{~N} \mathrm{NaOH}) ;$ lit. $^{15}[\alpha]_{\mathrm{D}}{ }^{23}=+17(\mathrm{c}$ $=2, \mathrm{EtOH} / 2 \mathrm{~N} \mathrm{NaOH})$

$N$-Benzyloxycarbonyl-(R)- $\alpha$-aminoadipic acid $\alpha$-benzyl ester; Cbz-(R)-Aad(OH)-OBzl (6). ${ }^{16}$ M.p $=91-93{ }^{\circ} \mathrm{C} ;$ lit. ${ }^{16} \mathrm{~m} . \mathrm{p}=90-92{ }^{\circ} \mathrm{C} .[\alpha]_{\mathrm{D}}{ }^{23}=+13.5(\mathrm{c}=1$, acetone);

$\boldsymbol{N}$-Benzyloxycarbonyl-(R)-pipecolic acid benzyl ester; Cbz-(R)-Pip-OBzl (7). $\mathrm{BH}_{3} \cdot \mathrm{Me}_{2} \mathrm{~S}(23$ $\mathrm{mL}$ of a $2 \mathrm{M}$ solution in THF, $46.0 \mathrm{mmol})$ was added to a solution of protected Cbz- $(R)-$ $\operatorname{Aad}(\mathrm{OH})-\mathrm{OBzl} 6(3.0 \mathrm{~g}, 8.49 \mathrm{mmol})$ in $70 \mathrm{~mL}$ of anhydrous THF at room temperature and the resulting mixture was stirred for $3 \mathrm{~h}$ (monitored by TLC). After completion of the reaction it was quenched by addition of $\mathrm{MeOH}(3 \mathrm{~mL})$ and the solvents were evaporated under reduced pressure. The residue which was then purified by flash chromatography $\left(\mathrm{Et}_{2} \mathrm{O} / \mathrm{PE} ; 3: 1\right)$ to afford $1.71 \mathrm{~g} \mathrm{Cbz-}(R)$-Pip-OBzl $(63 \%)$ as pale yellow oil. $[\alpha]_{\mathrm{D}}{ }^{23}=+7.4\left(\mathrm{c}=1, \mathrm{CHCl}_{3}\right) .{ }^{1} \mathrm{H}$ NMR $(500$ $\mathrm{MHz}, \mathrm{CDCl}_{3}$, conformer mixture): $\delta=7.39-7.35(\mathrm{~m}, 10 \mathrm{H}, \mathrm{Ar}-\mathrm{H}), 5.23-4.89\left(\mathrm{~m}, 5 \mathrm{H}, \mathrm{Bn}_{-} \mathrm{CH}_{2}\right.$, $\mathrm{CH})$, 4.18-4.09 (m, $\left.1 \mathrm{H}, \mathrm{CH}_{2}\right), 3.14-3.00\left(\mathrm{~m}, 1 \mathrm{H}, \mathrm{CH}_{2}\right), 2.32-2.25\left(\mathrm{~m}, 1 \mathrm{H}, \mathrm{CH}_{2}\right)$ 1.76-1.63 (m, $\left.3 \mathrm{H}, \mathrm{CH}_{2}\right), 1.48-1.44\left(\mathrm{~m}, 1 \mathrm{H}, \mathrm{CH}_{2}\right), 1.31-1.24\left(\mathrm{~m}, 1 \mathrm{H}, \mathrm{CH}_{2}\right) .{ }^{13} \mathrm{C}$ NMR $\left(125.7 \mathrm{MHz}, \mathrm{CDCl}_{3}\right.$,

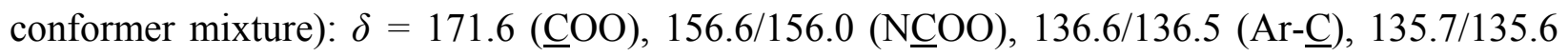
(Ar- $\underline{\mathrm{C}}), 127.8(10 \mathrm{Ar}-\underline{\mathrm{C}} \mathrm{H}), 67.3\left(\mathrm{Bn}-\underline{\mathrm{CH}_{2}}\right), 66.8\left(\mathrm{Bn}-\underline{\mathrm{CH}}_{2}\right), 54.7(\mathrm{CH}), 41.9 / 41.8\left(\underline{\mathrm{CH}}_{2}\right), 31.6$ 
/26.8 $\left(\underline{\mathrm{CH}}_{2}\right), 24.7 / 24.5\left(\underline{\mathrm{C}} \mathrm{H}_{2}\right), 20.7 / 20.6\left(\underline{\mathrm{CH}}_{2}\right) . \mathrm{C}_{21} \mathrm{H}_{23} \mathrm{NO}_{4}$ (353.41). HRMS (MALDI-FT-ICR): $m / z=376.15134$; calcd. for $\left[\mathrm{C}_{21} \mathrm{H}_{23} \mathrm{NO}_{4} \mathrm{Na}\right]^{+}: \mathrm{m} / z=376.15193$.

(R)-Pipecolic acid; H-(R)-Pip-OH (4). A solution of compound Cbz-(R)-Pip-OBzl 7 (414 mg, $1.17 \mathrm{mmol})$ in dry EtOH $(25 \mathrm{~mL})$ was mixed with $\mathrm{Pd} / \mathrm{C}(10 \%)(248 \mathrm{mg})$ and $\mathrm{AcOH}(0.3 \mathrm{~mL})$ in a hydrogenation flask and then the mixture was vigorously shaken at ambient temperature for 24 $\mathrm{h}$. Then the mixture was filtered through a pad of Celite. After the evaporation of the solvent under vacuum, the solid residue was dissolved in a little amount of $\mathrm{MeOH}$ and precipitated with $\mathrm{Et}_{2} \mathrm{O}$. The precipitate was collected by filtration, washed with $\mathrm{Et}_{2} \mathrm{O}$ and dried under high vacuum to afford pure $(R)$-pipecolic acid $4(132 \mathrm{mg}, 88 \%)$. All the physical data match the reported values. ${ }^{20} \mathrm{~m} . \mathrm{p}=271-273{ }^{\circ} \mathrm{C} ;$ lit. $^{20} \mathrm{~m} . \mathrm{p}=271-274{ }^{\circ} \mathrm{C} .[\alpha]_{\mathrm{D}}{ }^{23}=+26.0(\mathrm{c}=1$, water $) ;$ lit. $^{20}[\alpha]_{\mathrm{D}}{ }^{23}=$ $+26.3(\mathrm{c}=1$, water $)$.

(R)-2-(Benzyloxycarbonylamino)-6-hydroxyhexanoic benzyl ester $(\mathbf{8}) \cdot{ }^{17}$ Yield $=72 \%$; $[\alpha]_{\mathrm{D}}{ }^{23}$ $=+5.3\left(\mathrm{c}=1, \mathrm{CHCl}_{3}\right)$.

(R)-Benzyl 2-benzyloxycarbonyl-1,2,3,4-tetrahydropyridine-2-carboxylate (9). ${ }^{17}$ Yield = $420 \mathrm{mg}(56 \%) ;[\alpha]_{\mathrm{D}}{ }^{23}=+4.2\left(\mathrm{c}=1, \mathrm{CHCl}_{3}\right)$. The ${ }^{1} \mathrm{H}$ NMR spectrum matches reported data. ${ }^{17}{ }^{13} \mathrm{C}$ NMR (125.7 MHz, $\mathrm{CDCl}_{3}$, conformer mixture): $\delta=170.8 / 170.6\left(\underline{\mathrm{CO}}_{2} \mathrm{CH}_{2}\right), 153.4 / 153.2$

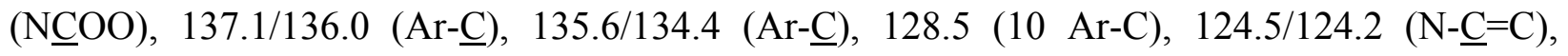
105.8/105.4 (N-C=ㅡ), 67.7/67.0 $\left(\mathrm{CO}_{2} \underline{\mathrm{CH}}_{2}\right), 66.9 / 66.8\left(\mathrm{CO}_{2} \underline{\mathrm{CH}}_{2}\right), 54.1 / 53.8(\mathrm{CH}), 23.5 / 23.4$ $\left(\mathrm{CH}_{2}\right), 18.4 / 18.2\left(\mathrm{CH}_{2}\right)$.

(R)-Benzyl 1-benzyloxycarbonyl-5-formyl-1,2,3,4-tetrahydropyridine-2-carboxylate (3). Phosphorus oxychloride $(0.39 \mathrm{~mL}, 4.27 \mathrm{mmol})$ was added dropwise to DMF $(0.33 \mathrm{~mL}, 4.27$ $\mathrm{mmol}$ ) at $10-20^{\circ} \mathrm{C}$ over a period of $3 \mathrm{~min}$ and the mixture was then stirred for $20 \mathrm{~min}$. After the mixture was cooled to $5{ }^{\circ} \mathrm{C}, 1,2$-dichloroethane $(3.0 \mathrm{~mL})$ was added, and then a solution of 9 $(150 \mathrm{mg}, 0.42 \mathrm{mmol})$ in 1,2-dichloroethane $(5 \mathrm{~mL})$ was added during $30 \mathrm{~min}$. The solution was stirred at $0-5^{\circ} \mathrm{C}$ for $1 \mathrm{~h}$ and was then refluxed for $15 \mathrm{~min}$. A solution of sodium acetate trihydrate $(70 \mathrm{mg})$ in water $(3.0 \mathrm{~mL})$ was added to the cooled mixture. The mixture refluxed for $15 \mathrm{~min}$ and then cooled to room temperature. The organic layer was separated and the aqueous phase was extracted with $\mathrm{CH}_{2} \mathrm{Cl}_{2}(3 \times 15 \mathrm{~mL})$. The combined organic phases were dried over anhydrous magnesium sulfate. After the drying agent was filtered off, the residue was purified by column chromatography on silica gel ( $n$-hexane/AcOEt; $1: 1)$ to give aldehyde 3 as pale yellow oil $(119 \mathrm{mg}, 73 \%) \cdot[\alpha]_{\mathrm{D}}{ }^{23}=+24.9\left(\mathrm{c}=1.1, \mathrm{CHCl}_{3}\right) .{ }^{1} \mathrm{H}$ NMR $\left(500 \mathrm{MHz}, \mathrm{CDCl}_{3}\right.$, conformer mixture): $\delta=9.35 / 9.26$ (s, $1 \mathrm{H}, \mathrm{CHO}$ ), 7.94/7.77 (s, $1 \mathrm{H}, \mathrm{CH}), 7.42-7.36$ (m, $10 \mathrm{H}, \mathrm{Ar}-\mathrm{H}), 5.36-$

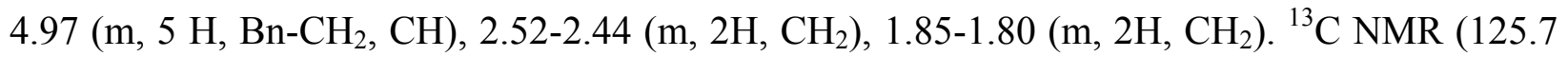

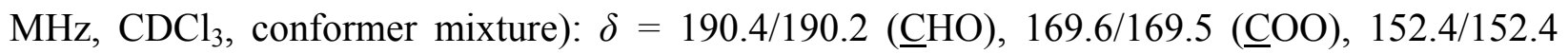

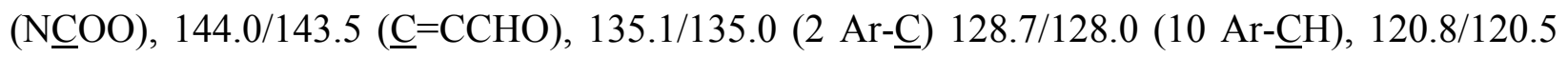
$(\mathrm{C}=\underline{\mathrm{CCHO}}), \quad 69.4 / 69.3 \quad\left(\mathrm{Bn}-\mathrm{CH}_{2}\right), 67.5 / 67.4 \quad\left(\mathrm{Bn}_{-}-\mathrm{CH}_{2}\right), \quad 55.3 / 54.9 \quad(\underline{\mathrm{CH}}), 22.5 / 22.3 \quad\left(\underline{\mathrm{CH}}_{2}\right)$ 15.4/15.1 $\left(\underline{\mathrm{CH}}_{2}\right) . \mathrm{C}_{22} \mathrm{H}_{21} \mathrm{NO}_{5}$ (379.41). HRMS (ESI-FT-ICR): $\mathrm{m} / \mathrm{z}=380.14921$; calcd. for $\left[\mathrm{C}_{22} \mathrm{H}_{22} \mathrm{NO}_{5}\right]^{+}: m / z=380.14925$.

(R)-Benzyl 1-benzyloxycarbonyl-5-vinyl-1,2,3,4-tetrahydropyridine-2-carboxylate (10). $n$ Butyl lithium $(0.1 \mathrm{~mL}, 1.6 \mathrm{M}$ in hexane, $0.08 \mathrm{mmol})$ was added to a stirred solution of 
triphenylmethylphosphonium bromide $(79 \mathrm{mg}, 0.08 \mathrm{mmol})$ in THF $(5 \mathrm{~mL})$ and the resulting mixture was stirred at room temperature for $30 \mathrm{~min}$. The solution of aldehyde $3(25 \mathrm{mg}, 0.07$ $\mathrm{mmol})$ in THF $(3 \mathrm{~mL})$ was added dropwise to the mixture. The reaction mixture was stirred at room temperature for $1.3 \mathrm{~h}$. After completion of the reaction as monitored by TLC (hexane:EE, $7: 3$ ), it was quenched by addition of water and extracted with ether. The combined ether layers were dried and concentrated to give crude product which was then purified by flash chromatography on silica gel ( $n$-hexane/AcOEt; 7:3) to give product as colorless oil (15.5 mg, $60 \%) \cdot[\alpha]_{\mathrm{D}}{ }^{23}=+43.1\left(\mathrm{c}=1, \mathrm{CHCl}_{3}\right) ;{ }^{1} \mathrm{H} \mathrm{NMR}\left(500 \mathrm{MHz}, \mathrm{CDCl}_{3}\right.$, conformer mixture): $\delta=7.42-$ 7.35 (m, 10H, Ar-H), 7.10/6.98 (s, 1H, CH), 6.41-6.27 (m, 1H, CH), 5.30-5.12 (m, 4H, Bn-CH $)$, 5.04-4.97 (m, 1H, CH), 4.92-4.87 (m, 1H, CH), 4.18-4.03 (m, 1H, CH), 2.50-2.40 (m, 1H, $\left.\mathrm{CH}_{2}\right)$, 2.30-2.23 (m, $\left.1 \mathrm{H}, \mathrm{CH}_{2}\right), 2.03-1.86\left(\mathrm{~m}, 1 \mathrm{H}, \mathrm{CH}_{2}\right), 1.60-1.50\left(\mathrm{~m}, 1 \mathrm{H}, \mathrm{CH}_{2}\right) .{ }^{13} \mathrm{C} \mathrm{NMR}(125.7$ $\mathrm{MHz}, \mathrm{CDCl}_{3}$, conformer mixture): $\delta=170.6 / 170.4$ (ㅁO), 153.3/153.1 (NCOO), 136.7/136.6

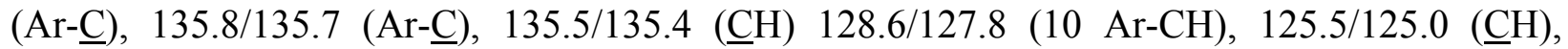
117.6/117.2 (므), 109.3/109.1 $\left(\underline{\mathrm{CH}}_{2}\right), 68.5 / 68.2\left(\mathrm{Bn}-\underline{\mathrm{CH}}_{2}\right), 67.6 / 67.0\left(\mathrm{Bn}-\underline{C H}_{2}\right), 54.2 / 53.9(\underline{\mathrm{CH}})$ 30.5/29.7 $\left(\underline{\mathrm{CH}}_{2}\right), 23.2 / 23.0\left(\underline{\mathrm{CH}}_{2}\right) . \mathrm{C}_{23} \mathrm{H}_{23} \mathrm{NO}_{4}$ (377.43). HRMS (MALDI-FT-ICR) $\mathrm{m} / z=$ 400.15204; calcd. for $\left[\mathrm{C}_{23} \mathrm{H}_{23} \mathrm{NO}_{4} \mathrm{Na}\right]^{+}: m / z=400.15193$.

(R)-Ethyl 6-chloro-5-formyl-1,2,3,4-tetrahydropyridine-2-carboxylate (11). A solution of anhydrous $N, N$-dimethylformamide $(0.24 \mathrm{~mL}, 2.90 \mathrm{mmol})$ in dry chloroform $(2.40 \mathrm{~mL})$ was added dropwise to a stirred solution of phosphorus oxychloride $(2.90 \mathrm{mmol}, 0.44 \mathrm{~mL})$ under a nitrogen atmosphere at room temp. After $30 \mathrm{~min}$, a solution of 6-oxopipecolic acid ethyl ester $\mathbf{2 b}$ (100 mg, $1.80 \mathrm{mmol})$ in $5 \mathrm{~mL}$ of dry chloroform was added. After $18 \mathrm{~h}$ stirring at room temperature, a solution of sodium acetate $(70 \mathrm{mg})$ dissolved in water $(5 \mathrm{~mL})$ was slowly added. After $0.5 \mathrm{~h}$, the mixture was partitioned between water and chloroform, and the aqueous phase was extracted with ethyl acetate. The organic phases were mixed and dried with anhydrous magnesium sulfate. The organic solvent was removed in vacuum and the residue was then purified by flash chromatography ( $n$-hexane-AcOEt; 7:3). The product was obtained in the form of a pale yellow oil $(88 \mathrm{mg}, 70 \%) .[\alpha]_{\mathrm{D}}{ }^{23}=+2.1\left(\mathrm{c}=0.5, \mathrm{CHCl}_{3}\right) ;{ }^{1} \mathrm{H} \mathrm{NMR}(500 \mathrm{MHz}$, $\mathrm{CD}_{3} \mathrm{COCD}_{3}$, conformer mixture): $\delta=8.98 / 8.03$ (s, $\left.1 \mathrm{H}, \mathrm{CHO}\right), 5.27-5.24(\mathrm{~m}, 1 \mathrm{H}, \mathrm{CH}), 4.20$ (q, $\left.2 \mathrm{H}, J=7.5, \mathrm{CH}_{2}\right), 2.43-2.37\left(\mathrm{~m}, 1 \mathrm{H}, \mathrm{CH}_{2}\right), 2.27-2.20\left(\mathrm{~m}, 1 \mathrm{H}, \mathrm{CH}_{2}\right), 2.16-2.11\left(\mathrm{~m}, 1 \mathrm{H}, \mathrm{CH}_{2}\right)$, 1.98-1.92 (m, 1H, $\left.\mathrm{CH}_{2}\right), 1.25\left(\mathrm{t}, 3 \mathrm{H}, J=7.5 \mathrm{~Hz}, \mathrm{CH}_{3}\right) .{ }^{13} \mathrm{C} \mathrm{NMR}\left(125.7 \mathrm{MHz}, \mathrm{CDCl}_{3}\right.$, conformer

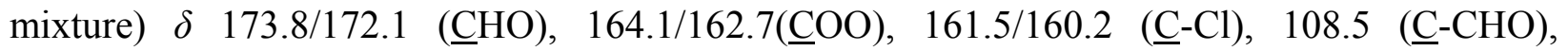
62.1/62.0 (ㅌCOO), 54.7/53.8 $\left(\underline{\mathrm{CH}}_{2}\right), 30.2 / 29.7\left(\underline{\mathrm{CH}}_{2}\right), 24.9 / 22.7\left(\underline{\mathrm{CH}}_{2}\right), 15.2 / 14.1\left(\underline{\mathrm{CH}}_{3}\right)$. $\mathrm{C}_{9} \mathrm{H}_{12} \mathrm{ClNO}_{3}$ (217.65). HRMS (EI): $m / z=217.04930$; calcd.: $m / z=217.05057$.

(R)-Methyl 6-thioxopiperidine-2-carboxylate (12a). 6-Oxopipecolic acid methyl ester 2 a (56.9 $\mathrm{mg}, 0.12 \mathrm{mmol}$ ) was placed in a round bottom flask, the system was purged with anhydrous nitrogen and the compound was dissolved in anhydrous toluene $(2.0 \mathrm{~mL})$. Lawesson's reagent $(45.0 \mathrm{mg}, 0.11 \mathrm{mmol})$ was added to this solution. Once the reagent was added, the reaction mixture was stirred at room temperature for $15 \mathrm{~min}$ and then three hours at $90{ }^{\circ} \mathrm{C}$. After the completion of reaction (monitored by TLC) the solvent was removed under reduced pressure. The residue was then subjected to column chromatography ( $n$-hexane/DCM; 7:3) to give pure 
product 12a as yellow oil $(41 \mathrm{mg}, 80 \%) \cdot[\alpha]_{\mathrm{D}}{ }^{23}=+10.8\left(\mathrm{c}=1, \mathrm{CHCl}_{3}\right) ;{ }^{1} \mathrm{H}$ NMR $(500 \mathrm{MHz}$, $\left.\mathrm{CDCl}_{3}\right): \delta=8.48$ (br.s, $\left.1 \mathrm{H}, \mathrm{NH}\right), 4.12(\mathrm{~m}, 1 \mathrm{H}, \mathrm{CH}), 3.83\left(\mathrm{~s}, 3 \mathrm{H}, \mathrm{CH}_{3}\right), 2.98\left(\mathrm{~m}, 1 \mathrm{H}, \mathrm{CH}_{2}\right), 2.88$ $\left(\mathrm{m}, 1 \mathrm{H}, \mathrm{CH}_{2}\right), 2.28\left(\mathrm{~m}, 1 \mathrm{H}, \mathrm{CH}_{2}\right), 1.88\left(\mathrm{~m}, 2 \mathrm{H}, \mathrm{CH}_{2}\right), 1.78\left(\mathrm{~m}, 1 \mathrm{H}, \mathrm{CH}_{2}\right) ;{ }^{13} \mathrm{C} \mathrm{NMR}(125.7 \mathrm{MHz}$, $\left.\mathrm{CDCl}_{3}\right) \delta=203.8(\mathrm{CS}), 169.9(\mathrm{COO}), 56.4(\mathrm{CH}), 53.1\left(\mathrm{COOCH}_{3}\right) 39.0\left(\mathrm{CH}_{2}\right), 24.1\left(\mathrm{CH}_{2}\right), 19.4$ $\left(\mathrm{CH}_{2}\right) . \mathrm{C}_{7} \mathrm{H}_{11} \mathrm{NO}_{2} \mathrm{~S}$ (173.23). HRMS (EI): $m / z=173.05070$; calcd.: $m / z=173.05105$.

$(\boldsymbol{R})$-Ethyl 6-thioxopiperidine-2-carboxylate (12b). The ethyl derivative was obtained from $\mathbf{2 b}$. Yield: $70 \mathrm{mg}, 56 \%$. $[\alpha]_{\mathrm{D}}{ }^{23}=+11.7\left(\mathrm{c}=1, \mathrm{CHCl}_{3}\right) ;{ }^{1} \mathrm{H} \mathrm{NMR}\left(500 \mathrm{MHz}, \mathrm{CDCl}_{3}\right): \delta=8.45(\mathrm{~s}, 1 \mathrm{H}$, $\mathrm{NH}), 4.36-4.28\left(\mathrm{~m}, 2 \mathrm{H}, \mathrm{CH}_{2}\right), 4.07(\mathrm{~m}, 1 \mathrm{H}, \mathrm{CH}), 3.00\left(\mathrm{~m}, 1 \mathrm{H}, \mathrm{CH}_{2}\right), 2.87\left(\mathrm{~m}, 1 \mathrm{H}, \mathrm{CH}_{2}\right), 2.29(\mathrm{~m}$, $\left.1 \mathrm{H}, \mathrm{CH}_{2}\right), 1.87\left(\mathrm{~m}, 2 \mathrm{H}, \mathrm{CH}_{2}\right), 1.78\left(\mathrm{~m}, 1 \mathrm{H}, \mathrm{CH}_{2}\right), 1.33\left(\mathrm{t}, J=10 \mathrm{~Hz}, 3 \mathrm{H}, \mathrm{CH}_{3}\right) ;{ }^{13} \mathrm{C} \mathrm{NMR}(125.7$ $\left.\mathrm{MHz}, \mathrm{CDCl}_{3}\right) \delta=203.7(\mathrm{CS}), 169.4(\mathrm{COO}), 62.4\left(\mathrm{CH}_{2}\right), 56.5(\mathrm{CH}), 39.0\left(\mathrm{CH}_{2}\right), 24.2\left(\mathrm{CH}_{2}\right), 19.4$ $\left(\mathrm{CH}_{2}\right), 14.1\left(\mathrm{CH}_{3}\right) . \mathrm{C}_{8} \mathrm{H}_{13} \mathrm{NO}_{2} \mathrm{~S}$ (187.26). HRMS (MALDI-FT-ICR) $\mathrm{m} / \mathrm{z}=210.05582$; calcd. for $\left[\mathrm{C}_{8} \mathrm{H}_{13} \mathrm{NO}_{2} \mathrm{SNa}\right]^{+}: m / z=210.05592$.

(R)-Diethyl 2-(6-(methoxycarbonyl)-piperidin-2-ylidene)malonate (13). Diethyl bromomalonate $(0.2 \mathrm{~mL}, 0.33 \mathrm{mmol})$ was added to a solution of thiolactam 12a $(51 \mathrm{mg}, 0.27 \mathrm{mmol})$ in $\mathrm{CH}_{2} \mathrm{Cl}_{2}(5 \mathrm{~mL})$ and the resulting mixture was stirred for $2 \mathrm{~h}$. Triethylamine $(0.05 \mathrm{~mL}, 0.33$ mmol) was added and the solution was stirred for two more hours. It was then diluted with $\mathrm{CH}_{2} \mathrm{Cl}_{2}$ and washed with $1 \mathrm{M}$ aqueous $\mathrm{HCl}$, the aqueous phases were extracted with $\mathrm{CH}_{2} \mathrm{Cl}_{2}$, the combined organic phase was dried, evaporated and the residue was purified by flash chromatography ( $n$-hexane/AcOEt 7:3) to give 13 as yellow oil $(72 \mathrm{mg}, 72 \%) \cdot[\alpha]_{\mathrm{D}}{ }^{23}=+3.0(\mathrm{c}=$ $\left.1, \mathrm{CHCl}_{3}\right) ;{ }^{1} \mathrm{H}$ NMR $\left(500 \mathrm{MHz}, \mathrm{CDCl}_{3}\right): \delta=4.22-4.19\left(\mathrm{~m}, 4 \mathrm{H}, \mathrm{COOC}_{2}\right), 4.17(\mathrm{~m}, 1 \mathrm{H}), 3.80(\mathrm{~s}$, $\left.3 \mathrm{H}, \mathrm{COOC}_{3}\right), 2.67\left(\mathrm{~m}, 2 \mathrm{H}, \mathrm{CH}_{2}\right), 2.22\left(\mathrm{~m}, 1 \mathrm{H}, \mathrm{CH}_{2}\right), 1.89-1.79\left(\mathrm{~m}, 3 \mathrm{H}, \mathrm{CH}_{2}\right), 1.32-1.26(\mathrm{~m}$,

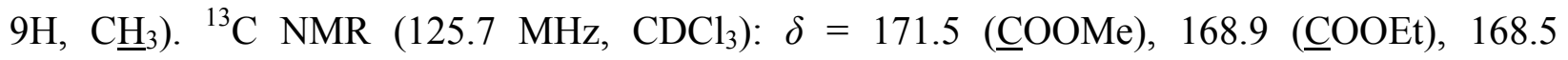
$(\underline{\mathrm{COOEt}}), 163.5(\mathrm{~N}-\underline{\mathrm{C}}=\mathrm{C}), 91.6(\mathrm{~N}-\mathrm{C}=\underline{\mathrm{C}}), 60.3(\mathrm{CH}), 59.5\left(\mathrm{COO}^{\mathrm{C}} \mathrm{H}_{2} \mathrm{CH}_{3}\right), 53.7\left(\mathrm{COO}^{-} \mathrm{H}_{2} \mathrm{CH}_{3}\right)$, $52.7\left(\mathrm{COOCH}_{3}\right), 26.6\left(\mathrm{CH}_{2}\right), 25.0\left(\mathrm{CH}_{2}\right), 18.2\left(\mathrm{CH}_{2}\right), 14.3\left(\mathrm{CH}_{3}\right) 14.2\left(\mathrm{CH}_{3}\right) . \mathrm{C}_{14} \mathrm{H}_{21} \mathrm{NO}_{6}$ (299.32). HRMS (ESI-FT-ICR): $m / z=322.12587$; calcd. for $\left[\mathrm{C}_{14} \mathrm{H}_{21} \mathrm{NO}_{6} \mathrm{Na}\right]^{+}: m / z=322.12611$.

\section{Acknowledgements}

The authors gratefully acknowledge the German Academic Exchange Service DAAD for the award of a PhD fellowship to Amina Sadiq. Sandoz GmbH, Kundl, Austria, and Trend Materials $\mathrm{GmbH}$, Linz, Austria, supported the project by providing the starting material $(R)$ - $\alpha$-aminoadipic acid.

\section{References}

1. Hughes, A. B., Ed. Amino Acids, Peptides and Proteins in Organic Chemistry Wiley-VCH: Weinheim, 2009.

2. Royo Gracia, S.; Gaus, K.; Sewald, N. Future Med. Chem. 2009, 1, 1289. 
3. Sonawane, V. C. Crit. Rev. Biotechnol. 2006, 26, 95.

4. Couty, F. Amino Acids 1999, 16, 297.

5. Nakatsuka, M.; Ragan, J. A.; Sammakia, T.; Smith, D. B.; Uehling, D. E.; Schreiber, S. L. J. Am. Chem. Soc. 1990, 112, 5583.

6. Gatto, G. J., Jr.; Boyne, M. T., $2^{\text {nd }}$; Kelleher, N. L.; Walsh, C. T. J. Am. Chem. Soc. 2006, $128,3838$.

7. Germann, U. A.; Shlyakhter, D.; Mason, V. S.; Zelle, R. E.; Duffy, J. P.; Galullo, V.; Armistead, D. M.; Saunders, J. O.; Boger, J.; Harding, M. W. Anticancer Drugs 1997, 8, 125.

8. Boger, D. L.; Chen, J.-H.; Saionz, K. W. J. Am. Chem. Soc. 1996, 118, 1629.

9. Fujii, T.; Mukaihara, M.; Agematu, H.; Tsunekawa, H. Biosci. Biotechnol. Biochem. 2002, 66,622 .

10. Singh, S. B.; Zink, D. L.; Liesch, J. M.; Mosley, R. T.; Dombrowski, A. W.; Bills, G. F.; Darkin-Rattray, S. J.; Schmatz, D. M.; Goetz, M. A. J. Org. Chem. 2002, 67, 815.

11. Bieliauskas, A. V.; Pflum, M. K. H. Chem. Soc. Rev. 2008, 37, 1402.

12. Zhang, X.; Chen, D.; Liu, X.; Feng, X. J. Org. Chem. 2007, 72, 5227.

13. Cowell, S. M.; Lee, Y. S.; Cain, J.P.; Hruby, V. J. Curr. Med. Chem. 2004, 11, 2785.

14. Kadouri-Puchot, C.; Comesse, S. Amino Acids, 2005, 29, 101.

15. Claesen, M.; Vlietinck, A.; Vanderhaeghe, H. Bull. Soc. Chim. Belg. 1968, 77, 587.

16. Baldwin, J. E.; Herchen, S. R.; Johnson, B. L.; Jung, M.; Usher, J. J.; Wan, T. J. Chem. Soc., Perkin Trans. I 1981, 2253.

17. Tice, C. M.; Ganem, B. J. Org. Chem. 1983, 48, 5043.

18. Huang, S.-B.; Nelson, J. S.; Weller, D. D. Synth. Commun. 1989, 19, 3485.

19. Pabba, J.; Mohal, N.; Vasella, A. Helv. Chim. Acta, 2006, 89, 1373.

20. Watanabe, L. A.; Haranaka, S.; Jose, B.; Yoshida, M.; Kato, T.; Moriguchi, M.; Soda, K.; Nishino N. Tetrahedron: Asymm. 2005, 16, 903. 